\title{
Use of alivecor heart monitor for heart rate and rhythm evaluation in dairy water buffalo calves (Bubalis Bubalis)
}

\begin{abstract}
Smartphone-based ECG recording devices are a promising new diagnostic in veterinary medicine. However, these devices have not yet been demonstrated to work in water buffalo. A commercial, non-invasive, smartphone-based ECG (AliveCor Vet TM) monitoring device was field tested on water buffalo calves. Six neonatal water buffalo calves had an ECG simultaneously recorded with a standard medical ECG recorder and the AliveCor Vet device. A correlation of 0.75053 was observed between average heart rates obtained between AliveCor recordings when compared to those from the 6 lead ECG. $100 \%$ Rhythm agreement was met for all 12 recordings. The recordings of 4/6 calves most closely resembled lead aVR, recordings of $2 / 6$ calves more closely resembled lead II. No significant difference was observed between standard and AliveCor quality scores $(\mathrm{P}=0.3632)$. We conclude that the AliveCor device allows for accurate rate diagnosis, and accurate diagnosis of a normal sinus rhythm in neonatal diary water buffalo calves in a field setting.
\end{abstract}

Keywords: buffalo, electrocardiogram, calves, smartphone, alivecor
Volume 4 Issue 2 - 2016

\author{
Joe Smith,' Jessica Ward, ${ }^{2}$ Tara Urbano, ${ }^{3}$ \\ Mikaela Mueller ${ }^{4}$ \\ 'Veterinary Diagnostic and Production Animal Medicine, lowa \\ State University, USA \\ ${ }^{2}$ lowa State University College of Veterinary Medicine, USA \\ ${ }^{3}$ University of California-Davis, USA \\ ${ }^{4}$ Blue Pearl Veterinary Partners, USA
}

Correspondence: Joe Smith, Veterinary Diagnostic and Production Animal Medicine, lowa State University, 1809 S Riverside Drive Ames, IA 500 I I, USA, Tel 7243554300 Email jss303@iastate.edu

Received: August 05, 2016 | Published: November II, 2016

\section{Introduction}

Research into the application of electrocardiography in buffaloes has been lacking when compared to other species. However, identification of altered health states, whether for identification of healthy animals or for the measurement of treatment effects is vital for research. Several electrocardiogram (ECG) changes were shown to occur in buffaloes naturally infected with Theileria annulata including atrial premature beat, sinus tachycardia, sinus arrhythmia, and first degree AV block. ${ }^{1}$ Similarly, significant changes in ECG amplitudes and intervals can be seen in buffaloes undergoing medetomidinebutorphanol and midazolam-butorphanol premedication for anesthesia. ${ }^{2}$ Arrythmias such as wandering pacemaker and sinus tachycardia have been reported in buffaloes undergoing ketamine anesthesia, ${ }^{3}$ demonstrating the need for ECG monitoring of bubaline surgical patients. Instrumentation that can allow for easy investigation of cardiac parameters is lacking for water buffaloes, as currently only traditional stand-alone ECG units have been reported in the literature.

An exciting new biotechnology is the use of commercial smartphones as medical devices. With recent advances in smartphone technology more medical techniques are accessible through one's cellular phone. One of the more recently developed devices is the AliveCor ECG device (AliveCor). This device allows a smartphone user to use their phone for the collection of heart rate, and rhythm in the form of an electrocardiogram that can be instantly accessed as well as recorded for later evaluation. The product described use focuses on canine, feline, and equine patients. In addition to determination of average heart rate, the device can be used to identify sinus rhythm, atrial fibrillation and ventricular premature complexes, ventricular pre-excitation and asynchronous depolarization of ventricles ${ }^{4}$ by a trained veterinarian. In some cases the device is replacing the traditional Holter monitor for ECG monitoring due to its portability and ease of use. ${ }^{4}$ There are reports in the literature of the device's use in a wide variety of animals including pigs ${ }^{5}$ Harbor Seal pups ${ }^{6}$ and a Savanah Monitor.

In the present study, we evaluated the AliveCor device for use in assessing the heart rate and rhythm in neonatal water buffalo calves. We hypothesized that the AliveCor would accurately record heart rates compared to standard 6-lead ECG, and that any rhythm abnormalities would be evident on both devices. By quality-scoring and evaluating the data we establish support for the use of the AliveCor device for the collection of data and as an adjunct to the physical examination of neonatal water buffalo calves by the established technique.

\section{Methods}

\section{Animals}

Six healthy neonatal commercial dairy water buffalo calves. Three male and three female calves were used for this study. Ages ranged from 3 to 17days of age (mean 9days).

\section{Data collection}

Calves were evaluated with AliveCor ECG recordings and standard 6 lead ECG recordings simultaneously obtained for each calf. The AliveCor device was placed in the region of the left heart base, caudal and medial to the elbow. The skin of the left heart base was clipped and ultrasound gel was utilized for better skin contact with the device. Paper speed was $25 \mathrm{~mm} / \mathrm{sec}$ and amplitude was $10 \mathrm{~mm} / \mathrm{mV}$. To mimic field settings all recordings were taken with the calf standing and haltered. The AliveCor device was held in a vertical orientation in the region medial to the left elbow. The same AliveCor Veterinary heart monitor and iPhone 5S (Apple inc, Cupertino, CA, USA) were used for each calf. Placement of the device is highlighted in Figure 1. Standard 6-lead ECGs were collected with a standard 6-lead electrocardiogram recorder (MAC-1200, Marquette Hellige GmbH, Germany). 




Figure I Placement of the AliveCor Vet device. The AliveCor device was placed in the dashed region on the left axillary aspect of the calf.

\section{Data evaluation}

All AliveCor and standard readings were evaluated unpaired and independently by a data-blinded veterinary cardiologist. The average heart rate was determined based on 6 seconds of ECG recording for both the AliveCor and the standard ECG. Additionally, the AliveCor calculated rate was recorded. A rhythm diagnosis was recorded for each. The findings were then reviewed to determine if the AliveCor device was comparable to the 6 lead ECG for rate and rhythm diagnosis. All leads were quality scored utilizing a previously reported ECG quality scoring system as depicted in Table $1 .{ }^{8}$ An example of a standard ECG compared to an AliveCor ECG is displayed in Figure 2.

Table I ECG quality scoring system as reported by Stern et al. ${ }^{8}$

\begin{tabular}{ll} 
Score & Description \\
\hline 0 & $\begin{array}{l}\text { High-quality recording with no baseline wander or small } \\
\text { baseline deflections }\end{array}$ \\
I & $\begin{array}{l}\text { Intermittent, mild tremors Or baseline deflections or } \\
\text { mild baseline wander }\end{array}$ \\
& $\begin{array}{l}\text { Moderate tremors or baseline deflection consistent } \\
\text { throughout the recording }\end{array}$ \\
3 & $\begin{array}{l}\text { Severe tremor artifact inhibiting the interpretation of the } \\
\text { P and T waves }\end{array}$
\end{tabular}

\section{Statistical analysis}

A Bland-Altman (Tukey Means-Difference) analysis was used to assess for significant difference between each calf's average heart rate determined by the AliveCor device, the AliveCor reading as determined by the cardiologist, and the 6-lead ECG. ECG quality scores were compared via a matched pair's $t$ test. This was done with a commercially available software program (JMP Pro 12, SAS; Cary, $\mathrm{NC}, \mathrm{USA}$ ). A p value of less than 0.05 was considered statistically significant for all values.



Figure 2 Standard ECG (left) compared to same segment on the AliveCor ECG (Right). Both recordings were gathered at settings of $25 \mathrm{~mm} / \mathrm{s}$ and $10 \mathrm{~mm} /$ $\mathrm{mV}$.

\section{Results}

There was a modest correlation between average heart rates obtained between AliveCor recordings when compared to those from the 6 lead ECG (0.75053) as determined by the Bland-Altman (Tukey Means-Difference) analysis (Figure 3). For this analysis the AliveCor heart rate averaged 111.667 beats/minute, and the standard ECG averaged 116.667 beats/minute. Standard error was 4.36697 and $P$ was 0.3070 . A normal sinus rhythm was recorded for all 12 recordings. As such all standard ECGs agreed with their AliveCor counterparts. The AliveCor recordings of $4 / 6$ calves most closely resembled lead aVR, recordings of $2 / 6$ calves more closely resembled lead II. No significant difference was observed between standard and AliveCor quality scores when compared via a matched-pairs t-test $(\mathrm{P}=0.3632)$. All calves tolerated standing AliveCor collection with minimal discomfort.

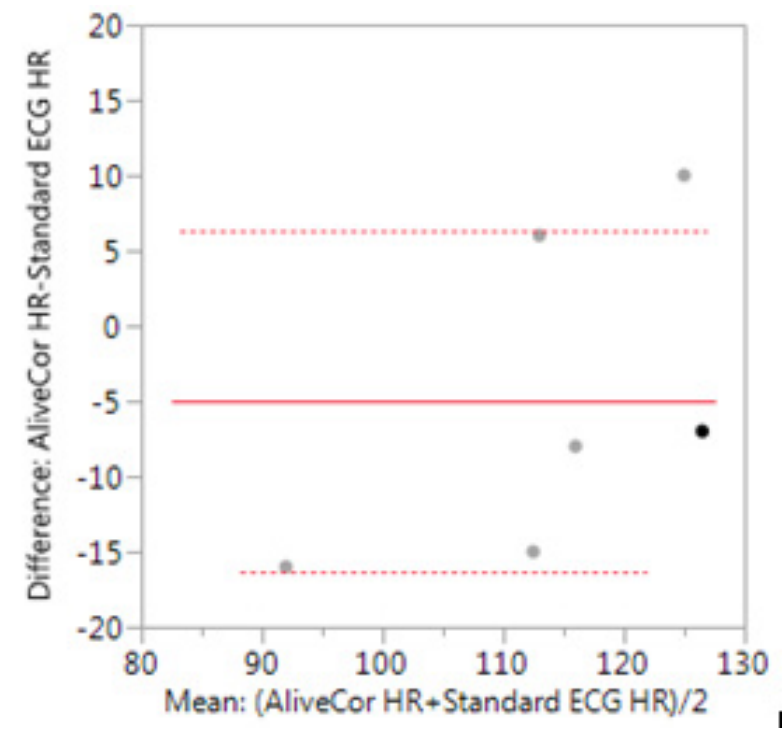

Figure 3 Bland Altman Plot of AliveCor HR and Standard ECG HR.

\section{Discussion}

As we hypothesized, the AliveCor allowed accurate heart rate recordings in all calves and allowed accurate diagnosis of sinus rhythm. Furthermore, the AliveCor device was easy to use, produced usable tracings in all calves, in an easily recordable format that would serve well for the collection of research data or as an adjunct to physical examination of water buffalo calves.

This agreement in sinus rhythm is encouraging, and rhythm 
agreement has been noted in several human studies with the device. ${ }^{9,10}$ The AliveCor recordings of $4 / 6$ calves most closely resembled lead aVR, recordings of $2 / 6$ calves more closely resembled lead II. This is in contrast to other species, as the device has most closely resembled lead II in cats, ${ }^{11}$ and in one report it most commonly resembled lead I in people. ${ }^{12}$ The standard ECG leads in bovine calves are also different from standard 6-lead ECG in dogs, cats, and people in terms of mean electrical axis. This is due to bovine species having a completely different Purkinje system compared to small animals and humans, where their entire ventricle depolarizes at nearly exactly the same time. Due to physical similarity to bovine calves a similar electrical axis presentation could be expected for bubaline calves.

In human medicine the device has been used for multiple applications. For patients with atrial fibrillation, no difference was found in corrected QT interval for AliveCor intervals or standard ECG recorded intervals. ${ }^{9}$ Similarly the AliveCor device has been used to supraventricular tachycardia in pediatric patients ${ }^{13}$ and has been shown to generate tracings of diagnostic quality in children. ${ }^{10}$ The cost-effective nature of this device has allowed for accurate detection of baseline intervals, atrial rate and rhythm when used for screening in a diverse human population. ${ }^{14}$ The device has also been shown to be used to successfully monitor college athletes experiencing heart palpitations for potentially adverse arrhythmias in real-time. ${ }^{15}$ Future implications have been made for the evaluation of acute ischemia with the device in people. ${ }^{16}$ There appear to be many future applications for this device in water buffaloes.

This study had several limitations. These results were collected from a relatively small sample size, and age range, from one herd. While the goal of the study was to evaluate the AliveCor device in calves, the study was performed in animals within a relatively small age window. Interestingly, in our study, we found no difference in quality scores between AliveCor tracings and standard 6-lead tracings. However, this may be partly because of positioning as the study was done with the calves standing, and based on results from other species, more agreement in heart rate and quality scores could have been reached from placing the calves in a right lateral recumbent position. In dogs, standing recordings has been shown to induce artifact on recordings in the form of muscle tremors, sporadic muscle twitching, and gross muscle movement. ${ }^{8}$ This same study has shown that a right lateral recumbent position improves the quality of ECG recordings in dogs by decreasing muscle tremor artifact, and shifting the mean electrical axis to the right relative to standing. ${ }^{8}$ Future research for this device includes the use of the device on animals in a right lateral recumbent position, older animals, as well as anesthesia monitoring. Similarly, the use of the device to record and be utilized to diagnose abnormal ECGs warrants further exploration.

\section{Conclusion}

We conclude that the AliveCor device allows for accurate heart rate diagnosis, and accurate diagnosis of a normal sinus rhythm in neonatal diary water buffalo calves in field setting conditions when used on the left heart base. Applications for this device include an adjunct to physical examination and recording of vital parameters in buffaloes for both research and medical purposes.

\section{Authors' contributions}

JS executed the experiment protocol, recorded data, blinded the data, analyzed the results and drafted the manuscript. MM and TU performed protocol design and data collection. JW determined heart rate, heart rhythm, and assigned quality scores to the blinded data. All authors approved of the final manuscript.

\section{Acknowledgements}

None.

\section{Conflict of interest}

Author declares that there is no conflict of interest.

\section{References}

1. Hasanpour A, Moghaddam GA, Nematollahi A. Biochemical, hematological, and electrocardiographic changes in buffaloes naturally infected with Theileria annulata. Korean $J$ Parasitol. 2008;46(4):223-227.

2. Malik V, Kinjavdekar P, Amarpal, et al. Comparative evaluation of halothane anaesthesia in medetomidine-butorphanol and midazolam-butorphanol premedicated water buffaloes (Bubalus bubalis). JS Afr Vet Assoc. 2011;82(1):8-17.

3. Pathak SC, Nigam JM, Peshin PK, et al. Anesthetic and hemodynamic effects of ketamine hydrochloride in buffalo calves (Bubalus bubalis). Am J Vet Res. 1982;43(5):875-878.

4. Brloznik M, Avbelj V. Wireless electrocardiographic monitoring in veterinary medicine. 2015 38th International Convention on Information and Communication Technology, Electronics and Microelectronics (MIPRO). 2015:356-359.

5. Madariaga ML, Michel SG, Tasaki M, et al. Induction of cardiac allograft tolerance across a full MHC barrier in miniature swine by donor kidney cotransplantation. Am J Transplant. 2013;13(10):2558-2566.

6. Fonfara S, D Casamian-Sorrosal, J Sundermeyer, et al. Variations in heart rate and rhythm of harbor seal pups during rehabilitation. Marine Mammal Science. 2014;31(3):998-1013.

7. Cushing AC, RJ Ossiboff, SE Knafo, et al. Coelomic and Pericardial Effusion Associated with Mesothelioma in a Savannah Monitor (Varanus exanthematicus). Journal of Herpetological Medicine and Surgery. 2014;24(3-4):66-71.

8. Stern JA, Hinchcliff KW, Constable PD. Effect of body position on electrocardiographic recordings in dogs. Aust Vet J. 2013;91(7):281-286.

9. Chung EH, Guise KD. QTC intervals can be assessed with the AliveCor heart monitor in patients on dofetilide for atrial fibrillation. J Electrocardiol. 2015;48(1):8-9.

10. Nguyen HH, Van Hare GF, Rudokas M, et al. SPEAR Trial: Smartphone Pediatric ElectrocARdiogram Trial. PLoS One. 2015;10(8):e0136256.

11. Mueller M, Orvalho J. Use of Alivecor Heart Monitor for Rate and Rhythm Evaluation in Cats. Journal of Veterinary Internal Medicine. 2013:644-644 p.

12. Baquero GA, Banchs JE, Ahmed S, et al. Surface 12 lead electrocardiogram recordings using smart phone technology. $J$ Electrocardiol. 2015;48(1):1-7.

13. Ferdman DJ, Liberman L, Silver ES. A Smartphone Application to Diagnose the Mechanism of Pediatric Supraventricular Tachycardia. Pediatr Cardiol. 2015;36(7):1452-1457.

14. Haberman ZC, Jahn RT, Bose R, et al. Wireless Smartphone ECG Enables Large-Scale Screening in Diverse Populations: J Cardiovasc Electrophysiol. 2015;26(5):520-526. 
15. Peritz DC, Howard A, Ciocca M, et al. Smartphone ECG aids real time diagnosis of palpitations in the competitive college athlete. J Electrocardiol. 2015;48(5):896-899.
16. Muhlestein JB, Le V, Albert D, et al. Smartphone ECG for evaluation of STEMI: results of the ST LEUIS Pilot Study. J Electrocardiol. 2015;48(2):249-259. 